\title{
TRATAMENTO DE ACNE VULGAR COM O USO DE ISOTRETINOINA
}

\author{
Lays de Brito de Pontes ${ }^{1}$ \\ Lívia Cabral Lobo ${ }^{2}$
}

RESUMO: Acne vulgar é uma doença cutânea muito rotineira, acometendo 85 a I0o\% dos indivíduos em determinada fase da vida, é uma dermatose de alta predominância, notadamente em adolescentes e adultos jovens. Esta patologia é habitualmente fragmentada em três tipos clínicos: comedônica, pápulo - pustulosa e nódulo - cística. O diagnóstico é clínico, contudo se obtêm várias alternativas terapêuticas farmacológicas permissíveis, dentre elas destaca-se a Isotretinoína, cuja utilização deve ser cautelosa, tendo em consideração as suas indicações.

Palavras-chave: Acne Vulgar. Isotretinoína. Tratamento. Atenção Farmacêutica.

ABSTRACT: Acne vulgaris is a very routine cutaneous disease, affecting 85 to $100 \%$ of those produced during life. It is a highly prevalent dermatosis, especially in adolescents and young adults. This pathology is usually divided into three clinical types: comedonic, papule - pustular and nodule - cystic. The diagnosis is clinical, however if you obtain several permissible pharmacological therapeutic alternatives among them, Isotretinoin stands out. Use should be cautious, taking into account its indications.

Keywords: Acne Vulgaris. Isotretinoin. Treatment. Pharmaceutical attention.

\section{INTRODUÇÃO}

A acne vulgar ou acne juvenil é uma afecção de pele que abrange a unidade pilossebácea. É uma doença de origem genética, não contagiosa, cujas ocorrências consistem da presença dos hormônios sexuais, é uma das doenças de pele mais frequentes (BIESKI, 20I6).

As lesões geralmente originam na puberdade, acometendo aproximadamente $80 \%$ dos adolescentes. Notamos que na acne vulgar a idade de implicação na maior

\footnotetext{
I Graduação em Farmácia, Nova Iguaçu-RJ, Brasil. E-mail: laysbrito58@gmail.com.

${ }^{2}$ Msc. em Ciências de Tecnologia de Processos Químicos e Bioquímicos pela Universidade Federal do Rio de Janeiro (UFRJ). Professora assistente no Departamento de Farmácia da Universidade Iguaçu (UNIG).
} 
parte das ocorrências é no período da adolescência apresentando-se em especial na face e no tronco, em algumas regiões do corpo com grande quantidade de glândulas sebáceas. Os sintomas variam de pessoa para pessoa, sendo, na maioria das vezes de curta e média intensidade (FLÁVIA, et al., 2013.).

Ao analisar a isotretinoína oral, pode acarretar inúmeros efeitos colaterais e tem vasto potencial teratogênico. Por conseguinte o tratamento para acne tem de se indicado a situações mais graves (graus III, IV e V) e quando há uma objeção a outras terapias. Inclusive em 1940 não se encontrava terapia satisfatória contra a acne, aplicavam-se tratamentos tópicos praticamente ineficientes ou apenas esperavam a cura natural. Em 1955 foi substanciada a isotretinoína e usufruída pela primeira vez em 1976 na Europa e em 1980 nos Estados Unidos para o tratamento de acne grave (NETO et al., 2015).

De acordo com Nascimento et al., (2011), ao contrário do que muitos acreditam no Brasil, foi aplicada pela primeira vez em 1982, tornando-se então um medicamento renovador na terapia contra acne grave e persistente devido aos seus abundantes mecanismos de ação que inclui a redução do tamanho da glândula sebácea, cancelamento de sua atividade e a diminuição de sebo, além do resultado imunológico e anti-inflamatório e da erradicação do Propionibacterium acnes no folículo pilossebáceo. Posto que a isotretinoína venha sendo consumida há décadas, resultando melhorias às pessoas com acne (SILVA et al.,, 2014).

\section{OBJETIVOS}

\section{I Objetivo Geral}

Discorrer sobre o tratamento da acne vulgar utilizando a isotretinoína, mencionado os seus benefícios no tratamento.

\subsection{Objetivos Específicos}

- Mencionar o que é Acne Vulgar;

- Descrever sobre a fisiopatologia da Acne Vulgar; 
- Explicar o como funciona o tratamento da Acne e o mecanismo de ação da isotretinoína;

- Listar os benefícios e as reações adversas da Isotretinoina;

- Explicar a importância da atenção farmacêutica no tratamento.

\section{METODOLOGIA}

A presente pesquisa foi feita através de revisão bibliográfica. Foram pesquisados artigos em bases periódicos como o Google Acadêmico e Biblioteca Virtual em Saúde (BVS), utilizando as palavras-chave: Acne Vulgar; Isotretinoína; Tratamento; Atenção Farmacêutica. Os textos utilizados, foram do período de 2010 á 2021, e outros quando se fez conveniente devido sua imensa importância para o trabalho.

\section{JUSTIFICATIVA}

Justifica-se a escolha do tema, pela prevalência de casos com acne vulgar e suas consequências psicossociais, caso o paciente não busque tratamento.

\section{ऽ REVISÃO DA LITERATURA}

\section{I ACNE VULGAR}

A acne é uma patologia frequente na população em geral, atingindo principalmente adolescentes e adultos jovens. É uma doença que se manifesta em vários graus de intensidade e, tendo a face como local de manifestação mais comum. Caracterizada por alteração fisiopatológica, a acne pode gerar problemas paralelos tais como: depressão, redução da autoestima, e até discriminação social (BRITO et al., 2010).

Diante do exposto, a acne em adolescentes é totalmente diferente, pois é possível encontrar o predomínio de pápulas e pústulas com total ausência de comedões. Antes identificados como uma erupção acneiforme e não como acne verdadeira, seu aparecimento em geral, estava relacionado a fatores externos como: o 
uso de drogas, cosméticos em demasia, produtos químicos, entre outros (BOUZAS \& MENESES, 2009).

FIGURA r: Acne vulgar grau III em mulher adulta

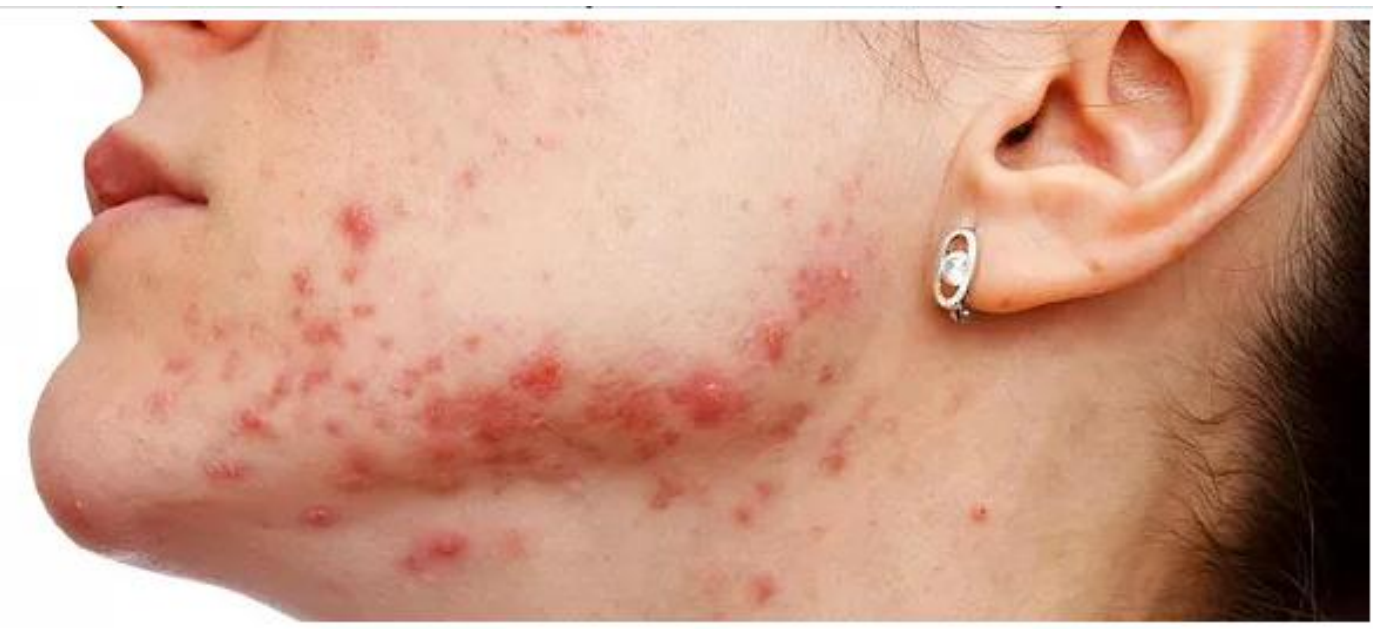

FONTE: Avallon, 2021.

É importante ressaltar que, a acne vulgar é a doença cutânea mais frequente que acomete de 85 a $100 \%$ da população em algum momento da vida. Sendo caracterizada por lesões que resultam da ação dos hormônios sobre as glândulas sebáceas da pele, afetando as áreas com maior densidade de folículos sebáceos (MENDES et al., 2016).

Segundo Silva et al., (2014) a acne é uma doença genético-hormonal, autolimitada, de localização pilossebácea com formação de comedões, pápulas e cistos, sua evolução soma um processo inflamatório de maior intensidade levando a formação de pústulas e abscessos, com frequente êxito cicatricial. Existem quatro fatores determinantes da acne: hipersecreção das glândulas sebáceas, alteração no processo de queratinização, colonização do folículo piloso por micro-organismos, nomeadamente Propionibacterium acnes e Staphylococcusalbuse liberação de mediadores inflamatórios na pele (KOLBE \& SILVA, 2017).

\subsection{FISIOPATOLOGIA DA ACNE}

De acordo com Neto et al., (2015), podemos notar que a acne é uma das dermatoses que mais acarreta incômodo social. Calcula-se que cerca de 40 a 50 milhões de pessoas vivenciem esta doença, caracterizando a desordem cutânea mais 
vista por dermatologistas. Visto que é uma doença genético-hormonal, autolimitada, de colocação pilossebácea, com desenvolvimento de comedões, pápulas e cistos, em cujo avanço se agrega processo inflamatório de maior veemência, estendendo à formação de pústulas e abscessos.

A pele que é responsável pela proteção, sensação, secreção e termorregulação, é um envoltório elástico que preserva o homem do meio ambiente em combate a passagem de agentes químicos e físicos, e interdita a eliminação exorbitante de água e eletrólitos (BIESKI, 2016).

Além disso, a camada subcutânea de células adiposas que se encontra sob a derme e além da fáscia muscular, não é tida como parte propriamente dita da pele, mas não se deve pular ao falar de sua anatomia (KOLBE \& SILVA, 2017).

Ainda convém lembrar que a unidade pilossebácea na pele normal é integrada por glândulas sebáceas multilobulares, pelo rudimentar e canal folicular abrigado de um epitélio escamoso estratificado. No entanto o avanço dessas unidades é conduzido por hormônios androgênicos e alguns outros fatores biológicos. Os hormônios correlacionados com a patogênese da acne são a de hidrotestosterona e testosterona, incumbidos por controlar a atividade das glândulas sebáceas (SILVA et al., 2014).

$\mathrm{Na}$ fase adulta, os fatores hormonais passam a ser atenuados, com consequente redução da ocorrência da acne. Porém, alguns autores evidenciam a influência de outros aspectos, como a ação de neuromediadores e hiperandrogenia, na persistência dos quadros de acne além do período de adolescência, bem como no surgimento tardio da doença (HASSUN, 2010).

As reações inflamatórias associadas na acne, de uma forma geral, acarretam na liberação de vários mediadores inflamatórios, culminando na ruptura da parede da glândula, reação responsável por essas lesões (NIJSTEN et al., 2010).

Essa manifestação cutânea pode aparecer em vários graus. Em certos casos, as lesões são mínimas, e não chegam a gerar incômodo ao paciente, ao passo de que em outros, elas podem se tornar tão evidentes, que perturbam a qualidade de vida, e desencadeiam ou agravam problemas emocionais e de autoestima, comprometendo a 
integridade psíquica. Conforme o grau de severidade e localização tem-se cinco formas, a I, II, III, IV e V (FAURE, 2010 \& ROMITI et al., 2010).

O tipo I, conhecido como acne comedônica, compreende então, os comedões, oleosidade da pele e poros dilatados, afetando principalmente a face. Existem três tipos conhecidos de comedões: o microcomedão, o comedão fechado, e o comedão aberto, onde este último é facilmente eliminado, porém, pode vir a apresentar inflamações. Já o tipo II, denominada de acne pápulo-pustulosa, esta se apresenta por pápulas e pústulas na face, com um quadro de intensidade variável, desde as poucas lesões, até as mais numerosas, com intensa inflamação. O grau seguinte é o III, chamado de nódulo-cística, onde aparecem nódulos e cistos. Além desses, também podem ser vistos os comedões, as papúlas, as pústulas e os nódulos furunculóides - cistos, formados a partir da reação inflamatória que atinge a profundidade do folículo até o pêlo, podendo ocorrer à formação de pus. O grau IV é a acne conglobata, uma forma mais grave, onde se somam os nódulos purulentos em grande número, formando até mesmo abscessos e fístulas. Normalmente neste grau, são verificadas lesões na face, colo e dorso (KROWCHUCK, 2008).

No entanto, a fase mais grave da doença é a acne fulminans - grau V. É bastante rara, e pode chegar a causar febre, leucocitose e poliartralgia. Embora a gravidade do quadro evolua do grau I para o grau V, por exemplo, pode ocorrer à somatória de graduação em diferentes níveis de acne no desenvolvimento dos quadros clínicos (ROMITI et al., 2010).

FIGURA 2: Tipos de acne

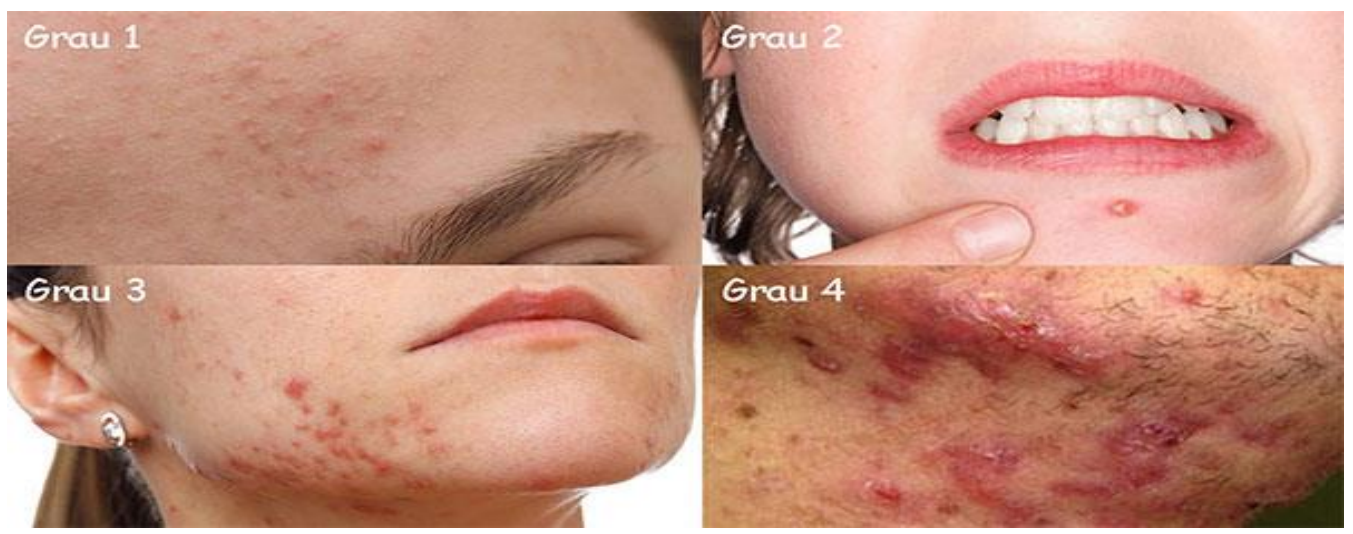

Fonte: Dermato Virual, 2020. 
Por outro lado, se ocorre uma superabundância de sebo elaborado pela glândula provoca uma oclusão na região do infidíbulo da unidade, podendo advir o desenvolvimento de um microcomedão, indicador de todas as lesões acnéicas. Com isso, tais danos também estão correlacionados a bactéria Propionibacterium acnes, que se hospedam na glândula sebácea e passa a usufruir dos triglicerídeos por ação enzimática, concedendo substâncias importunas que agem como quimiotáticos para as células de defesa do organismo, despertando pápulas, pústulas, cistos inflamatórios e nódulos (MCKOY, 2018).

\subsection{TRATAMENTO FARMACOLÓGICO DA ACNE}

O tratamento $\mathrm{da}$ acne pode ser feito por via oral ou por via tópica. $\mathrm{Na}$ atualidade, o tratamento mais comum para a acne de grau moderado a grave é feito com a isotretinoína oral. $\mathrm{O}$ tratamento da acne visa controlar os diferentes fatores que levam ao seu surgimento. Diante disso é possível identificar os objetivos principais da farmacoterapia dessa patologia, tratando-se de controlar a hiperceratose de retenção, reduzir a produção de sebo, diminuir a população bacteriana e eliminar a inflamação (KOLBE \& SILVA, 2017).

O tratamento da acne deve ser o mais precoce possível para evitar cicatrizes físicas e psicossociais, para tanto o uso da isotretinoína revolucionou o tratamento da acne. Desta forma pode-se afirmar que a terapia com isotretinoína dura em média de 4 a 6 meses, e só é observado a diminuição da acne após 2 meses de uso e o benefício do tratamento permanece por alguns meses após o termino da intervenção medicamentosa (BRASIL, 2015).

Os estudos apontam que os tratamentos tópicos e sistêmicos, que não a isotretinoína (antibióticoterapia e antiandrogênicos), para a acne vulgar nas mulheres costumam ser efetivos na melhora dos casos mais leves desta manifestação, principalmente quando os objetivos terapêuticos são os de corrigir as alterações metabólicas. Apesar disto o índice de recorrência e a dificuldade terapêutica quando relacionada a uma acne mais severa pode estar presente. Diante de tal constatação, a terapêutica da acne deve ser adequada à gravidade e morfologia das lesões sendo de suma importância o acompanhamento por um dermatologista e um farmacêutico 
auxiliando na prevenção e solucionando possíveis interações, e consequentemente monitorar e minimizar reações adversas ao fármaco da terapia medicamentosa (CARVALHO, 2005).

\subsection{ISOTRETINOÍNA}

A isotretinoína é o ácido 13 -cis-retinóico, um isômero sintético da tretinoína, administrado por via sistêmica. Medicamento da classe dos retinóides, que segundo Brito et al., (2010, p. 332), "são derivados sintéticos da vitamina A (retinol) e desenvolvem um papel fundamental, no tratamento da Acne, porque agem na lesão primária: no microcomedo, além de seu importante papel na supressão sebácea".

Sua utilização foi aprovada para uso em acne cística e nodular no ano de 1982 pelo Food and Drugs Administration (FDA), órgão norte americano responsável por proteger a saúde pública, garantindo a segurança e eficácia dos medicamentos de uso humano. Correspondendo a um dos fármacos com maior relevância no que diz respeito à história da dermatologia, possibilita, após sua inclusão como terapia na acne severa, uma real chance de cura aos pacientes, que anteriormente, já se encontravam fadados a tratamentos paliativos (NETO, 2015).

Mesmo que comprovadamente a droga ofereça alta eficácia, esta se confronta diretamente com seus efeitos adversos, onde a maioria ocasiona alterações metabólicas de alto grau, o que dificulta a conclusão do tratamento. A isotretinoína também possui inúmeras contraindicações, o que restringe seu uso, como no caso de mulheres grávidas, devido a um de seus principais efeitos, a teratogenicidade; indivíduos com problemas hepáticos, renais, hipervitaminose A, entre outras (BRASIL, 2015).

Há uma necessidade de conhecimento amplo por parte do dermatologista, quanto à prescrição da isotretinoína no tratamento da acne, onde as dosagens devem ser prescritas de maneira individualizada, conforme a gravidade do caso. Antes do início do tratamento o especialista, deverá informar ao usuário, os possíveis efeitos do fármaco, como também o monitoramento clínico-laboratorial através de teste de glicose, creatininaquinase, triglicerídeos, colesterol, funções hepáticas e possíveis efeitos oculares e psicológicos (PIANA \& CANTO, 20I0). 
Salienta-se a necessidade de que haja um controle rigoroso na dispensação desse medicamento, devido as suas peculiaridades, que com a prática da assistência farmacêutica, ocorrerá uma minimização dos riscos da administração da isotretinoína (CAJUEIRO et al., 2014).

\subsection{Farmacologia}

O mecanismo de ação da isotretinoína ainda não foi elucidado em pormenores, mas já se instituiu que a melhora observada no quadro clínico da acne grave para qual está recomendada, associa-se com a supressão dose-dependente da atividade da glândula sebácea e com a diminuição no tamanho das glândulas sebáceas, comprovada histologicamente. Estabeleceu-se, inclusive, o efeito antiinflamatório dérmico da isotretinoína (AZULAY \& AZULAY, 2008).

Porém, supostamente pode-se dizer que a isotretinoína é um fármaco que intervém de forma mais completa nos mecanismos fisiopatológicos da acne, através da redução da diferenciação sebácea, diminuição do tamanho da glândula sebácea e na sua capacidade de produção de sebo, além apresentar propriedades antiinflamatórias (AL-KHAWAJAH, 1996).

As doses comumente utilizadas variam entre $0,5-0,7 \mathrm{mg} / \mathrm{kg} / \mathrm{dia}$, em tomadas pós prandiais, de 6 a 8 meses, de forma a obter-se a dose total cumulativa de Ioo a I20 $\mathrm{mg} / \mathrm{kg}$. A terapêutica inicial com a isotretinoína é altamente eficaz, embora possa haver uma fase inicial de agravamento das lesões, especialmente nas formas com marcado componente retencional (AMICHAI; SHEMER; GRUNWALD, 20Io).

O ácido-I3-cis-retinóico evidencia grande eficiência terapêutica relacionada à indução e controle da diferenciação epitelial, nos tecidos secretores de muco ou queratinizantes, à produção de prostaglandinas E2, de colágeno, e de predecessores da queratina, como os tonofilamentos e tonofibrilas, e ao controle da proliferação de Propionibacterium acnes. As lesões inflamatórias associadas à acne estão estritamente relacionadas ao desenvolvimento das glândulas sebáceas e a exacerbação de sua atividade secretora, e assim, o bloqueio na produção de sebo provocado pela isotretinoína estabelece um fator decisivo na sua atividade farmacológica, e no tratamento dessa afecção (ALLEN \& BLOXHAM, 2оı; SAURAT, 2оıо). 
O perfil farmacocinético da isotretinoína é comparável com o da vitamina $\mathrm{A}$. Após a administração oral, o pico de concentração plasmática é atingido em cerca de 2 a 4 horas. Cerca de $20 \%$ da isotretinoína são absorvidos quando administrada com o estômago vazio, aumentando para $40 \%$ quando em presença de alimento. A isotretinoína e tretinoína são interconvertidas in vivo, e cerca de $20 \%$ a $30 \%$ da dose de isotretinoína são claramente metabolizadas nesta rota. $\mathrm{O}$ metabólito fundamental deste fármaco é a 4-oxo-isotretinoína, que é excretada pela bile após ser conjugada com o ácido glicurônico, apresentando meia-vida média de eliminação de 25 horas. Com a administração repetida, a concentração de equilíbrio ocorre de 5 a 7 dias (NANKERVIS et al., 2008; BRAZZELL \& COLBURN, 2007).

Alguns estudos farmacocinéticos efetuados em animais recebendo doses orais de isotretinoína marcada confirmaram que essa substância lipofílica possui distribuição tissular de 15 minutos, com máximo de I hora e declínio até níveis não detectáveis em um período de 24 horas em muitos tecidos, como por exemplo, o hepático, renal, adrenal, ovariano e glândulas lacrimais. A isotretinoína é excretada pela via urinária, detectando-se nesta a presença de quantidades baixas desse fármaco não conjugado. Porém, a excreção de cerca de 53 a $74 \%$ ocorre nas fezes, como resultado de uma absorção incompleta, eliminação biliar ou recirculação entreoepática (AHFS-DRUG INFORMATION, 2007; ALLEN \& BLOXHAM, 2010).

\subsection{BENEFÍCIOS DA ISOTRETINOÍNA}

A isotretinoína é considerado um retinóico natural e sintético de uso tópico e sistêmico, sendo única medicação que atua em todos os fatores etiopatogênicos da acne (RODRIGUES et al., 2019).

Os retinóides tem uma definição atual de conglomerar todos os compostos sintéticos e naturais ligados à da vitamina $\mathrm{A}$, são tópicos e considerados produtos excelentes para todas as formas de acnes, incluindo as inflamatórias, sua terapêutica inicial com a isotretinoína é considerado eficaz, podendo haver uma fase inicial de agravamento das lesões, especialmente nas formas com forte componente retencional (FIGUEIREDO et al., 2omb). 
De acordo com Zanelato, et al., (20II), pode ocorrer uma evolução da acne vulgar para acne fulminans que é mais rara, porém grave, o paciente inicia o tratamento com isotretinoína, cerca de duas semanas pode ocorrer piora do quadro com desenvolvimento de lesões úlcero-necróticas no tórax, face e dorso, além de febre e alterações laboratoriais. A melhora surgi após I a 2 meses ao inicio do tratamento, os resultados começam a aparecer na face e em seguida no tronco e seus benefícios permanecem alguns meses após o termino (BRASIL, 2015).

O tratamento reduz a produção do sebo e diminui o tamanho das glândulas, inibindo sua atividade e ajudando a normalizar a queratinização, restringindo o crescimento das acnes e reduzindo a inflamação. $O$ resultado final do tratamento é sempre eficaz, mesmo com algumas modificações hematológicas e bioquímicas, em pacientes com acne moderada e severa, tornando a isotretinoína um medicamento seguro e seus efeitos colaterais tolerados (BRITO et al., 2010).

\subsection{REAÇÕES ADVERSAS ASSOCIADAS AO USO DE ISOTRETINOÍNA ORAL E CONTRAINDICAÇÕES}

Apesar da sua efetividade no controle da acne, a isotretinoína tem apresentado vários efeitos adversos, como alterações mucocutâneos e efeitos tóxicos sistêmicos, no qual os mais comuns são: queilite, ressecamento da pele do corpo, aumento do colesterol, triglicerídeos e enzimas hepáticas. Portanto são necessários exames laboratoriais antes e durante o tratamento, afim de monitorar uma possível alteração no perfil lipídico, sendo esse um efeito colateral comum ao uso da isotretinoína. Estudo de corte populacional mostrou que a elevação de triglicerídeos ocorreu em $45 \%$ dos pacientes durante o tratamento, enquanto que aumento de colesterol total foi encontrado em 30\% deles (BRASIL, 2015).

Geralmente essas elevações são leves e não determinam a interrupção do tratamento. Os efeitos adversos causados pela utilização desse medicamento podem ser minimizados com sua redução, com exceção da teratogenicidade (CAJUEIRO et al., 2014).

Os efeitos adversos associados ao tratamento da acne com o uso da isotretinoína podendo ser divididos em duas classes: efeitos mucocutâneos e efeitos 
tóxicos sistêmicos. Usualmente, pacientes que usam isotretinoína oral manifestam ressecamento e fissuras nos lábios, pele e mucosas em vista da diminuição da produção de sebo. Além de reduzir a espessura do estrato córneo que provoca foto sensibilidade, blefaroconjuntivite, também é observado prurido, descamação da pele e dermatite (BRITO et al., 2010).

Kolbe \& Silva (2017) corroboram com o fato de que a isotretinoína é uma medicação segura e o único risco irreversível é, em mulheres, a ação teratogênica. É imprescindível que as mulheres realizem teste de gravidez antes de iniciar o tratamento com essa medicação. Incluem no grupo das contraindicações relativas ao uso da isotretinoína a obesidade com distúrbio de colesterol ou triglicérides; diabetes insulinodependente; doença hepática, renal ou sistêmica; epilepsia, depressão e psicose. Para o controle dessas condições devem ser realizados exames clínicos e laboratoriais mensais.

\subsection{ATENÇÃO FARMACÊUTICA}

A Atenção Farmacêutica é um modelo de prática farmacêutica desenvolvida no contexto da assistência farmacêutica. Ela compreende atitudes, valores éticos e compromissos na prevenção de doenças, promoção e recuperação da saúde de forma integrada à equipe de saúde. É a interação direta do farmacêutico com o paciente que visa promover uma melhora na qualidade de vida do paciente e fornecer orientações sobre o tratamento medicamentoso de modo a trazer benefícios para o paciente. A farmacoterapia adequada e a condição clínica e psicológica do paciente são elementos essenciais para o desenvolvimento dessa prática farmacêutica (PIANA; CANTO, 2010).

A pesquisa de Dutra et al., (2015) relatam a importância do acompanhamento farmacêutico e os benefícios que essa atenção farmacêutico-paciente pode trazer na vida do paciente; conservando a saúde e observando os possíveis agravamentos que podem surgir no tratamento. Diante disto, o farmacêutico deve interagir de forma ativa com o paciente de modo a resolver problemas que envolvam ou não o uso de medicamentos e acompanhar os seus resultados, para que desta forma, a dispensação 
do medicamento ao paciente seja feita de maneira eficaz, consciente e segura (PINHO et al., 2016).

O uso da Isotretinoína precisa ser realizado com a orientação de um farmacêutico para evitar possíveis consequências que pode acarretar durante o tratamento, e a melhor forma de informar aos pacientes sobre as precauções do uso deste medicamento é realizando a atenção farmacêutica. Assim, o farmacêutico se torna uma figura muito importante, pois é um profissional fundamental no ato da dispensação, tendo contato mais próximo em relação paciente-medicamento fornecendo informações sobre o uso correto (MOREIRA \& REGO, 20II).

Para Schmidt \& Silva (2013) O farmacêutico deve orientar o paciente com o uso da Isotretinoína, alertando sobre às interações medicamentosas, os cuidados na administração e conservação do medicamento, relatar sobre reações alérgicas (se for apresentada), outros medicamentos em uso, destacar a importância da realização correta do tratamento para obter um resultado eficaz, bem como acompanhar a realização de exames laboratoriais periódicos e o uso de medidas contraceptivas pelas pacientes.

\section{CONCLUSÃO}

A acne vulgar apresenta-se como uma doença dermatológica extremamente comum, que se manifesta em maior proporção em adolescentes e jovens adultos. Refere-se de um processo crônico decorrente de uma maior alteração de hormônios masculinos, que pode ocasionar lesões em tipos de comedões (cravos), pústulas, pápulas, e mais gravemente pode gerar cistos, abscessos e cicatrizes em graus variáveis.

Pela magnitude ocasionada pela acne vulgar, os indivíduos acometidos podem desenvolver reduções de autoestima, depressões, discriminações e estigmas sociais. Dentre a amplitude de problemas acarretados pela acne vulgar, a Isotretinoína apresenta-se como o tratamento mais eficaz. Apesar de o fármaco denotar uma boa efetividade, concomitantemente possui uma série de efeitos adversos expressivos que frequentemente dificulta o término do tratamento. 
O paciente usuário deve estar ciente, concordar e assinar os Termos de Esclarecimento e Regularidade a respeito do mesmo, manter as taxas glicêmicas controladas e estar atento a casos de gravidez, pelo fato da teratogenicidade.

Diante dos fatos expostos se faz necessário um tratamento e acompanhamento adequado com a participação de uma equipe multidisciplinar e inclusão relevante do farmacêutico, já que o mesmo é quem conhece a farmacologia do medicamento, seus efeitos adversos e interações.

\section{REFERÊNCIAS}

AHFS-DRUG INFORMATION. Skin and mucous membrane agents: miscellaneous skin and mucous membrane agents. Bethesda: AHFS, 2007.

ALLEN, J. G.; BLOXHAM, D. P. The pharmacology and pharmacokinetics of the retinoids. Pharmac. Ther., Oxford, v. 40, n. I, p. I-27, 2010.

AL-KHAWAJAH, M. M. Isotretinoin for acne vulgaris. Int. J. Dermatol., Philadelphia, v.35, n. 3, p. 212-215, 1996.

AMICHAI, B.; SHEMER, A.; GRUNWALD, M. H. Low-dose isotretinoin in the treatment of acne vulgaris. J Am Acad Dermatol, 2010.

AZULAY \& AZULAY. Dermatologia. 5 ed. Rio de Janeiro: Guanabara Koogan, 2008.

BIESK, Gustavo Luiz. Riscos e benefícios do uso do medicamento isotretinoína para - tratamento da acne. FACIDER Revista Científica. Mato Grosso, n 6, 2016. Disponível em: file:///E:/acne\%2ovulgar\%2oartigos/I49-588-I-PB.pdf. Acesso em: I8 de setembro de 2021.

BRITO, Maria de Fátima de Medeiros; et al., Avaliação dos efeitos adversos clínicos e alterações laboratoriais em pacientes com acne vulgar tratados com isotretinoína oral. An Bras Dermatol. Pernanbuco, v 3, n 85, 2010. Disponível em: file:///E:/acne\%2ovulgar\%2oartigos/ao6v85n3\%20(I)\%2obrito.pdf. Acesso em: 22 de setembro de 202I. 
BRASIL, Agência Nacional de Vigilância Sanitária. Protocolo de uso da isotretinoína no tratamento da acne grave, 2015.

BRAZZELL, R. K.; COLBURN, W. A. Pharmacokinetics of the retinoids isotretinoin and etretinate. J. Am. Acad. Dermatol., Saint Louis, 2007.

BRITO, M, F. M., et al. Avaliação dos efeitos adversos clínicos e alterações laboratoriais e, pacientes com acne vulgar tratados com isotretinoína oral. Anais Brasileiros de Dermatologia, v. 85, n. 3, p. 331-337, 2010.

CAJUEIRO, E. S.; LIMA, L. B. R.; PARTATA, A. K. Isotretinoína e suas propriedades farmacológicas. Revista Científica do ITPAC, jan, 2014.

CARVAlHO, L. J. A.; CARVAlHO, L. A. Benefícios da isotretinoína no tratamento da acne nódulo-cística em pacientes hiperandrogênicas devido à síndrome dos ovários policísticos. Revista Medicina Cutâneo Ibero Latino Americana, v. 33, n. 5, p. 21I-218, 2005.

DUAILIBE, Duailanne da Silva; ALVES, Nygell Silva. Isotretinoína - amplitude terapêutica e potencialidade tóxica. 6If. (Monografia de Bacharelado em Farmácia Bioquímica). 2007.

DUTRA, J. R.; SOUZA, S. M. F.; PEIXOTO, M. C. A influência dos padrões de beleza veiculados pela mídia, como fator decisório na automedicação com moderadores de apetite por mulheres no município de Miracema-RJ. Revista Transformar, $7^{\mathrm{a}}$ edição, 2015. Disponível em: http://www.fsj.edu.br/transformar/index.php/transformar/article/view/40/37.

Acesso em: i9 de setembro de 2021.

FAURE, M. Acné et hormones. Rev Prat. 2010; 52:850 -3.

FAVARIN, Flávia. Et al. O uso de isotretinoina oral no tratamento de acne vulgar. Revista Thêma et Scientia. Paraná, $\mathrm{v}$ 3, $\mathrm{n}$ 2, 2013. Disponível em: file:///E:/acne\%2ovulgar\%2oartigos/157-504-I-PB.pdf.Acesso em: 18 de setembro de 202I. 
FIGUEIREDO, Américo. Et al. Avaliação e tratamento do doente com acne - Parte II: Tratamento tópico, sistémico e cirúrgico, tratamento da acne na grávida, algoritmo terapêutico. Revista Portuguesa de Clínica Geral. Lisboa, 2orıb. disponível em: http://www.scielo.mec.pt/pdf/rpcg/v27nI/v27nıar2.pdf. Acesso em: 22 de setembro de 202I.

Figura I: Avallon, clínica dermatológica. Disponível em: https://www.clinicaavallon.com.br/acnefacialecorporal Acesso em: 23 de setembro de 2021.

Figura 2: Dermato Virtual. 2020. Disponível em: https://dermatovirtual.com.br/graus-etipos-de-acne-espinhas/ Acesso em: 23 de setembro de 2021.

HASSUN K. M. Acne: etiopatogenia. An Bras Dermatol. 75:7-I, 2010.

KROWCHUCK, D. P. Treating Acne. Med Clin North Am - Adolescent medicine. 2008: 8:8II-24.

KOLBE, Ana Christina; SILVA, Fernando Lima da. Uso da isotretinoína no tratamento da acne e sua relação com a halitose. Revista de Ciências Médicas e

Biológicas. Salvador, $\mathrm{v}$ I, $\mathrm{n}$ I6, 2017. Disponível em: file:///E:/acne\%2ovulgar\%2oartigos/ı4169-80670-I-PB.pdf. .Acesso em: i8 de setembro de 202I.

MCKOY, Karen. Acner Vulgar. Distúrbios dermatológicos. Estados Unidos, 2018. Disponível em: file:///E:/acne\%2ovulgar\%20artigos/Acne\%20vulgar\%20\%20Distúrbios\%20dermatológicos\%20\%20Manuais\%20MSD\%20edição\%20para\%20p rofissionais.pdf.Acesso em: 18 de setembro de 202I.

MOREIRA, G. C. Q.; RÊGO, V. R. P. DE A. Farmacoterapia da Isotretinoína Oral e Avaliação da Relação Risco/Benefício no Tratamento da Acne Vulgar. CRF-BA em revista Ano IV - № 17 - Dezembro/20II. Disponível em: http://www.crfba.org.br/revista/revista_crfba_17.pdf. Acesso em: I9 de setembro de 2021.

NANKERVIS, R. et al. Effect of lipid vehicle on intestinal lymphatic transport of isotretinoin in the rat. Int. J. Pharm., Amsterdam, 2008. 
NASCIMENTO, Carolina. Ribas. Et al .Recidiva de acne após tratamento com isotretinoína oral: seguimento de cinco anos. Surgical \& Cosmetic Dermatology. Bauru, v.3, n. 3, 20II. Disponível em: file:///C:/Users/Usuario/Downloads/v3Recidiva-de-acne-apos-tratamento-com-isotretinoina-oral--seguimento-de-cincoanos.pdf. Acesso em: 18 de setembro de 2021.

NETO, Edilson Martins Rodrigues. Et al. Abordagem terapêutica da acne na clínica farmacêutica. Boletim Informativo Geum. Ceará, 2015. Disponível em: file:///E:/acne\%2ovulgar\%2oartigos/3885-17346-2-PB.pdf. Acesso em: 18 de setembro de 202I.

NIJSTEN, T.; ROMBOUTS, S.; LAMBERT, J. Acne is prevalent but use of its treatments is infrequent among adolescents from the general population. J Eur Acad Dermatol Venereol 2010. Feb; 2I (2): 163-7

PIANA, M.; CANTO, S. G. Atenção Farmacêutica Em Dermatologia: Fármacos E Antiacneicos. Saúde (Santa Maria), 2oro. Disponível em https://periodicos.ufsm.br/revistasaude/article/viewFile/2488/1748. Acesso em: 19 de setembro de 202I.

PINHO , M. S.; ABREU , P. A.; NOGUEIRA, T. A. Atenção Farmacêutica a Pacientes Oncológicos: Uma Revisão Integrativa da Literatura. Scielo. 2016. Disponível em: www.sbrafh.org.br/rbfhss/public/artigos/201607010600o86oBR.pdf. Acesso em: I9 de setembro de 2021.

RODRIGUES, Fabiana Marques; et al., Tratamento dermatológico da acne vulgar. Revista saúde em foco. Itapetinga, São Paulo, n. II, 2019. Disponível em: file:///E:/acne\%2ovulgar\%2oartigos/o26_TRATAMENTO-DERMATOLÓGICODA-ACNE-VULGAR\%202019.pdf. Acesso em: 22 de setembro de 202I.

ROMITI, R.; JANSEN T.; PLEWIG. G. Acne fulminans. An bras dermatol 2oro; 75(5): 611617. 
SAURAT, J. H. Oral isotretinoin. Where now, where next? Dermatology, Basel, v. 195, n. I, p. I-3, 2010.

SCHMIDT, G. M.; SILVA, C. B. Isotretinoína: utilização no tratamento sistêmico da acne e aspectos fundamentais para a atenção farmacêutica. Revista Infarma, 2009. Disponível em: file://C:/Users/Windows\%208/Downloads/I46-539-IPB\%20(2).pdf. Acesso em: I9 de setembro de 2021.

SILVA, Ana margarida Ferreira da; COSTA, Francisco Pinto; MOREIRA, Margarida. Acne vulgar: diagnóstico e manejo pelo médico de família e comunidade. Revista Brasileira de medicina de família e comunidade. Rio de Janeiro, 2014. Disponível em: file:///E:/acne\%20vulgar\%20artigos/754-5197-I-PB.pdf. Acesso em: I8 de setembro de 202I.

ZANELATO, Tiago Pina. Et al. Acne fulminans incapacitante. Anais Brasileiros de Dermatologia. Rio de Janeiro, 20Ir. Disponível em: http://www.scielo.br/pdf/abd/v86n4si/v86n4siaor.pdf. Acesso em: 22 de setembro de 2021. 